\title{
Multi-Objective Optimization for Milling Operations using Genetic Algorithms under Various Constraints
}

\author{
Libao An* \\ College of Mechanical Engineering \\ Hebei Provincial Key Laboratory of Inorganic Nonmetallic Materials \\ Hebei United University \\ 46 Xinhua West Road, Tangshan, Hebei 063009, China \\ Email: lan@heuu.edu.cn \\ www.heuu.edu.cn \\ Peiqing Yang \\ Fushun Mechanical Equipment Manufacturing Co., Ltd \\ 50 Middle Section of Xincheng Road, Shuncheng District, Fushun, Liaoning 113006, China \\ Email: yangpeiqing2010@126.com \\ Hong Zhang \\ College of Life Sciences, Hebei United University \\ 57 Jianshe South Street, Tangshan, Hebei 063000, China \\ Email: zhanghong@heuu.edu.cn \\ Mingyuan Chen ${ }^{\dagger}$ \\ Department of Mechanical and Industrial Engineering \\ Concordia University \\ 1455 de Maisonneuve Blvd. W, Montreal, Quebec H3G 1M8, Canada \\ E-mail:mychen@encs.concordia.ca \\ www.concordia.ca
}

\begin{abstract}
In this paper, the parameter optimization problem for face-milling operations is studied. A multi-objective mathematical model is developed with the purpose to minimize the unit production cost and total machining time while maximize the profit rate. The unwanted material is removed by one finishing pass and at least one roughing passes depending on the total depth of cut. Maximum and minimum allowable cutting speeds, feed rates and depths of cut, as well as tool life, surface roughness, cutting force and cutting power consumption are constraints of the model. Optimal values of objective function and corresponding machining parameters are found by Genetic Algorithms. An example is presented to illustrate the model and solution method.
\end{abstract}

Keywords: face-milling operation; multi-objective optimization; machining parameter; genetic algorithms

\footnotetext{
*Address: 46 Xinhua West Road, Tangshan, Hebei 063009, China

${ }^{\dagger}$ Address: 1455 de Maisonneuve Blvd. W, Montreal, Quebec H3G 1M8, Canada
} 


\section{Introduction}

In today's manufacturing environment, many large companies in metal-cutting industries are making use of advanced manufacturing and management technologies to reduce production cost and increase profit. Machining parameter optimization plays an important role in meeting these requirements and it is an essential part of a Computer Aided Design/Computer Aided Process Planning/Computer Aided Manufacturing (CAD/CAPP/CAM) system. Machining parameter optimization usually involves the optimal selection of cutting speed, feed rate, depth of cut, and the number of passes. In practice, machining parameters are in most cases selected from machining database or handbooks. The cutting regimes given in such a way may not be the optimal values [1]. Single-objective optimization problems have been intensively studied using dynamic programming [2], geometric programming [3], linear programming [4], and some other techniques [5-6]. With the ever-increasing need for lowering cost and increasing production rate, several different and competing objectives have to be simultaneously optimized [7-9]. Multi-objective optimization problems have been studied since the early 1960s, especially during the past decade. The solutions for a multiobjective optimization problem may not meet all single objective functions and the obtained parameters cannot be simply compared with each other, and therefore the solutions are called non-dominated [10]. The existing models and processes for multi-objective optimization problems are usually complex and do not consider all practical constraints.

From a literature review, it can be known that machining parameter optimization has been performed mainly for turning process. Milling is a machining process of cutting material away by feeding a workpiece against a rotating cutter with multiple teeth. The machined surface may be a flat, angular, or curved one, or any combination of them, and thus milling is the most versatile machining process compared to the others such as turning, grinding, and reaming. In face milling, the cutter is mounted on a spindle rotating perpendicular to the machining surface. The cutting action of the many teeth on the periphery and face of the cutter forms the milled surface, providing a fast method of material removal. In this paper, the unit production cost, unit machining time and profit rate are optimized simultaneously for face-milling operations. A variety of realistic machining conditions and quality specifications are considered as constraints. The model is solved by Genetic Algorithms (GAs). An example is given to illustrate the model and solution procedure.

\section{Model Development}

Machining optimization models are mathematical programming models formulated from realistic machining processes. These models have objective functions based on certain economic criterion and subject to various practical constrains from machining conditions. In this section, a multi-objective machining optimizing model to minimize unit production cost and unit machining time as well as to maximize profit rate is proposed for multi-pass face-milling operations in single-tool applications. The total depth of material to be removed, including one finish pass and multiple rough passes, is cut with the same tool. Multi-pass machining operations are governed by complicated machining conditions.

\subsection{Objective function}

For a face-milling process, unit machining time TC (min) is comprised of actual machining time $t_{m}$ (min), machine idle time $t_{l}$ (min), and tool replacement time $t_{R}(\mathrm{~min})$. Dividing a milling process into one finish pass and $n$ rough passes, actual machining time $t_{m}=\frac{\pi D L_{t s}}{1000 V_{s} f_{s} Z}+\sum_{i=1}^{n} \frac{\pi D L_{t r}}{1000 V_{r i} f_{r i} Z}$, where $D(\mathrm{~mm})$ is the diameter of the cutter; $L_{t s}(\mathrm{~mm})$ is the finish cutting travel length, $L_{t s}=L+0.5\left(D-\sqrt{D^{2}-B^{2}}\right)+3 ; L_{t r}(\mathrm{~mm})$ is the rough cutting travel length, $L_{t r}=L+D+3 ; L(\mathrm{~mm})$ and $B$ $(\mathrm{mm})$ are respectively the length and width of the workpiece; $Z$ is the tooth number of the cutter; $V_{s}(\mathrm{~m} / \mathrm{min})$ and $f_{s}(\mathrm{~mm} /$ tooth$)$ are respectively cutting speed and feed rate for the finish pass; $V_{r i}(\mathrm{~m} / \mathrm{min})$ and $f_{r i}(\mathrm{~mm} /$ tooth) are respectively cutting speed and feed rate for the $i$-th rough pass. Machine idle time $t_{l}$ is defined as $t_{l}=t_{p}+t_{i}$ [2], where $t_{p}(\mathrm{~min})$ is preparation time, and $t_{i}$ (min) is idle tool motion time. Therefore, $t_{l}=t_{P}+n\left(h_{1} L_{t r}+h_{2}\right)+\left(h_{1} L_{t s}+h_{2}\right)$, where $h_{1}(\mathrm{~min} / \mathrm{mm})$ 
is tool travel time and $h_{2}$ (min) is tool approach/depart time. Tool replacement time $t_{R}$ can be given by $t_{R}=t_{e} Z \frac{t_{m}}{T}$, where $t_{e}$ (min) is tool exchange time, $T$ (min) is tool life. Then the objective function to minimize unit machining time can be written as Minimize:

$$
T C=t_{m}+t_{l}+t_{R}=t_{s}+\sum_{i=1}^{n} t_{r i}+t_{p},
$$

where

$$
\begin{aligned}
& t_{s}=\left(1+\frac{t_{e} Z}{T_{s}}\right) \frac{\pi D L_{t s}}{1000 V_{s} f_{s} Z}+\left(h_{1} L_{t s}+h_{2}\right), \\
& t_{r i}=\left(1+\frac{t_{e} Z}{T_{r i}}\right) \frac{\pi D L_{t r}}{1000 V_{r i} f_{r i} Z}+\left(h_{1} L_{t r}+h_{2}\right) .
\end{aligned}
$$

Similarly, unit production cost $U C(\$)$ is comprised of actual machining cost $C M$, machine idle cost $C I$, tool replacement cost $C R$, and tool cost $C T$, if material cost is not considered. $C M$ is based on actual machining time $t_{m}$ and labor cost, $k_{0}(\$ / \mathrm{min})$, including overhead, then $C M=k_{0} t_{m}$. The machine idle cost $C I$ is defined as $C I=k_{0} t_{l}$. Tool replacement cost $C R$ and tool cost $C T$ can be respectively given by $C R=k_{0} t_{R}$ and $C T=k_{t} Z \frac{t_{m}}{T}$, where $k_{t}(\$)$ is tool cost. Then

$$
U C=C M+C I+C R+C T=U C_{s}+\sum_{i=1}^{n} U C_{r i}+k_{0} t_{p}
$$

where

$$
\begin{gathered}
U C_{s}=\left(k_{0}+\frac{k_{t} Z}{T_{s}}+\frac{k_{0} t_{e} Z}{T_{s}}\right) \frac{\pi D L_{t s}}{1000 V_{s} f_{s} Z}+k_{0}\left(h_{1} L_{t s}+h_{2}\right), \\
U C_{r i}=\left(k_{0}+\frac{k_{t} Z}{T_{r i}}+\frac{k_{0} t_{e} Z}{T_{r i}}\right) \frac{\pi D L_{t r}}{1000 V_{r i} f_{r i} Z}+k_{0}\left(h_{1} L_{t r}+h_{2}\right) .
\end{gathered}
$$

The profit rate in face-milling process can be determined by [11]

$$
P_{t}=\frac{S_{p}-U C-C_{m a t}}{T C},
$$

where $S_{p}$ denotes the unit sale price of the product (\$), $C_{\text {mat }}$ represents the cost of raw material (\$).

\subsection{Constraints}

For given cutting conditions, there exist reasonable ranges of cutting speed, feed rate, and depth of cut, for either a finish or a rough pass:

$$
\begin{gathered}
V_{s, \text { min }} \leq V_{s} \leq V_{s, \text { max }}, f_{s, \text { min }} \leq f_{s} \leq f_{s, \text { max }}, d_{s, \text { min }} \leq d_{s} \leq d_{s, \text { max }}, \\
V_{r, \text { min }} \leq V_{r i} \leq V_{r, \text { max }}, f_{r, \text { min }} \leq f_{r i} \leq f_{r, \text { max }}, d_{r, \text { min }} \leq d_{r i} \leq d_{r \text {,max }} .
\end{gathered}
$$

Tool lives in face-milling can be given by

$$
T_{s}^{l}=\frac{C_{v} K_{v} D^{q_{v}}}{V_{s} d_{s}^{X_{v}} f_{s}^{y_{v}} B^{s_{v}} Z^{p_{v}}}, T_{r i}^{l}=\frac{C_{v} K_{v} D^{q_{v}}}{V_{r i} d_{r i}^{X_{v}} f_{r i}^{y_{v}} B^{S_{v}} Z^{p_{v}}},
$$

where $C_{v}, K_{v}, q_{v}, p_{v}, x_{v}, y_{v}$, and $s_{v}$ are constants; $T_{s}$ and $T_{r i}$ are tool lives (mm) in finish machining and rough machining, respectively. In this paper, we assume the tool lives are identical in finish and rough machining operations and require the same tool replacement time, i.e. $T_{s}=T_{r i}=T$. Surface finish requirements can by given by

$$
f_{s} \leq \sqrt{r_{e} R_{s, \max } / 0.0321}, f_{r i} \leq \sqrt{r_{e} R_{r, \text { max }} / 0.0321},
$$

where $r_{e}$ is cutter nose radius (mm); $R_{s, \text { max }}$ and $R_{r \text {,max }}$ are surface roughness requirements $(\mathrm{mm})$ for finish machining and rough machining, respectively. Cutting force constraints can be written as

$$
\begin{gathered}
F=\frac{C_{f} K_{f} B^{s_{f}} Z^{p_{f}} d_{s}^{x_{f}} f_{s}^{y_{f}}}{D^{q_{f}}} \leq F_{\max }, \\
F=\frac{C_{f} K_{f} B^{s_{f}} Z^{p_{f}} d_{r i}^{X_{f}} f_{r i}^{y_{f}}}{D^{q_{f}}} \leq F_{\max },
\end{gathered}
$$

where $C_{f}, K_{f}, x_{f}, y_{f}, p_{f}, q_{f}$ and $s_{f}$ are constants; $F_{\max }$ is maximum available cutting force (kgf). Cutting power can be derived by multiplying cutting force and cutting speed, 


$$
\begin{gathered}
P=\frac{F V_{s}}{6120 \eta}=\frac{C_{f} K_{f} B^{s_{f}} Z^{p_{f}} V_{s} d_{s}^{X_{f}} f_{s}^{y_{f}}}{6120 \eta D^{q_{f}}} \leq P_{\max }, \\
P=\frac{F V_{r i}}{6120 \eta}=\frac{C_{f} K_{f} B^{s_{f}} Z^{p_{f}} V_{r i} d_{r i}^{x_{f}} f_{r i}^{y_{f}}}{6120 \eta D^{q_{f}}} \leq P_{\max },
\end{gathered}
$$

where $\eta$ is the efficiency of the machine tool and $P_{\max }$ is the maximum power $(\mathrm{kW})$. The total depth of cut $d_{t}$ can be expressed as

$$
d_{t}=d_{s}+\sum_{i=1}^{n} d_{r i} .
$$

In the model, $V_{s}, f_{s}, d_{s}, V_{r i}, f_{r i}, d_{r i}$, and $n$ are decision variables.

\section{Solution Method}

The primary objectives in solving the machining parameter optimization problems are reliability, accuracy of results, and efficient computation. The selection of a suitable solution method for the optimization problem depends on the problem itself. The form and complexity of the objective functions and constraints influence the solution procedure to be applied. The solution approaches themselves have characteristics that affect their efficiency and accuracy. In this paper, the values of optimal unit production cost, optimal unit machining time, optimal profit rate and corresponding machining parameters are found by GAs as evolutionary algorithms are becoming more popular in engineering design due to their effectiveness, particularly in obtaining global optimal solutions.

\subsection{Solution procedure statement}

Genetic Algorithms is a particular class of evolutionary algorithms that make use of techniques motivated by evolutionary biology such as selection, mutation, and crossover. When solving the multi-objective optimization model using GAs, decision variables $V_{s}$, $f_{s}, d_{s}, V_{r i}, f_{r i}, d_{r i}$ are represented by binary numbers and these numbers are aligned in a long binary string which is called a chromosome. The population size in this research is 100 . The fitness function consists of the sum of the unit production cost, unit machining time and profit rate using different weight coefficients for each objective function: fit $=w_{1} U C+w_{2} T C+w_{3} P t$, where the values of weight coefficients can be decided based on the practical situation, and $w_{1}+w_{2}+w_{3}=1$. Crossover is the operation to exchange some part of two chromosomes to generate new offspring (crossover rate is $80 \%$ in this paper). This operation is important for exploring the whole search space rapidly. Mutation operation randomly alters each bit of a binary string after crossover with a small probability (mutation rate is 0.05 in this work) to provide a small uncertainty to the new chromosome. In the paper $20 \%$ chromosomes with best fitness values are kept within the population to avoid losing the best strings, and the rest chromosomes apply to a crossover or mutation operation during each reproduction cycle. The same population size is maintained during the evolution process. After crossover and mutation, a new generation forms and the values of objective functions and machining parameters are calculated. After a certain number of generations (2000 iterations in this research), the GA should converge to the best chromosome, which represents the optimal or near-optimal solution to the problem. Fig. 1 shows the diagram of the proposed genetic algorithm.

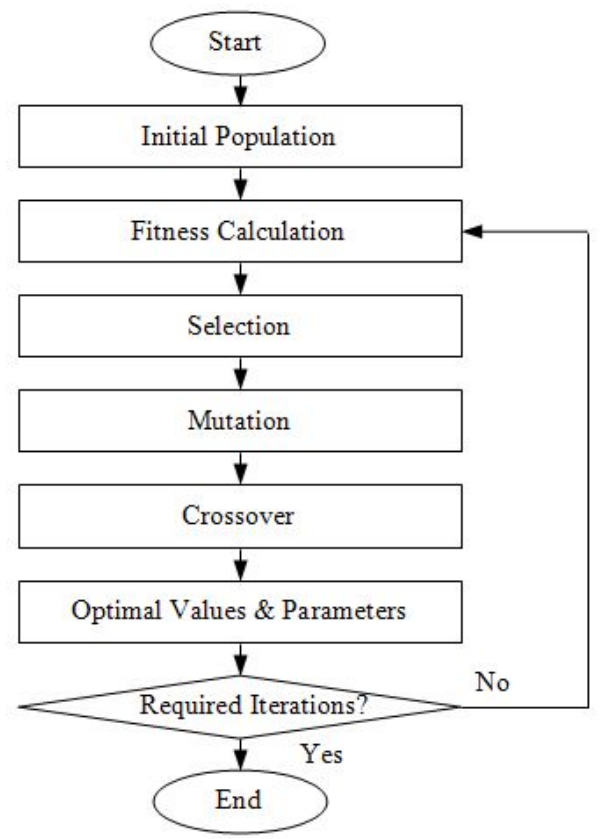

Fig. 1. GA flow chart

In this work, weight coefficients are respectively given by $w_{1}=0.6, w_{2}=0.2, w_{3}=0.2$. Based on a given value of the total depth of cut and the feasible ranges of rough and finish cutting passes, possible numbers of total passes can be calculated. The algorithm computes 
for each case and compares the results for an optimal pass number.

We assume that the unwanted material should be cut off with one finish pass and $n$ rough passes $(n \geq 1)$. Therefore, the total number of cutting passes is $N=n+1$. The total depth of cut considered in this paper is $2.0 \mathrm{~mm} \leq d_{t}<8 \mathrm{~mm}$.

When $n=1$, there are 5 decision variables: $V_{s}, V_{r}$, $f_{s}, f_{r}, d_{s}\left(d_{r}=d_{t}-d_{s}\right)$. When $n=2$, there are 8 decision variables: $V_{s}, V_{r 1}, V_{r 2}, f_{s}, f_{r 1}, f_{r 2}, d_{s}$, $d_{r 2}\left(d_{r 1}=d_{t}-d_{s}-d_{r 2}\right)$.

\section{2. $G A$ implementation for $n=1$}

We select $n=1$ as an example to explain how to use the genetic algorithm for solving solutions of the problem.

\subsubsection{Determination of the string length}

Before the initial population is generated, the total length of a binary string which represents cutting parameters in the given order needs to be determined based on the domain and precision of the decision variables.

(i) Cutting speed of the finish pass, $V_{s}$ : The parameter constraint is $50.0 \leq V_{s} \leq 300.0$. The domain of the variable is [50.0, 300.0] and the required precision is one place after the decimal point. The required number of bits for a binary variable can be calculated by $2^{L_{1}-1}<300.0 \times 10^{1} \leq 2^{L_{1}}-1$. Therefore, the length of a binary string for $V_{s}$ is $L_{1}=12$.

(ii) Cutting speed of the rough pass, $V_{r}$ : The domain of the variable is $[50.0,300.0]$ and the required precision is one place after the decimal point. Similarly, the length of a binary string for $V_{r}$ is $L_{2}=12$.

(iii) Feed rate of the finish pass, $f_{s}$ : The domain of the variable is $[0.10,0.60]$ and the required precision is two places after the decimal point. Therefore, we have $2^{L_{3}-1}<0.60 \times 10^{2} \leq 2^{L_{3}}-1$. The length of a binary string for $f_{s}$ is $L_{3}=6$.

(iv) Feed rate of the rough pass, $f_{r}$ : The domain of the variable is $[0.10,0.60]$ and the required precision is two places after the decimal point. Therefore, we have $2^{L_{4}-1}<0.60 \times 10^{2} \leq 2^{L_{4}}-1$. The length of a binary string for $f_{r}$ is $L_{4}=6$.

(v) Depth of cut for the finish pass, $d_{s}$ : The domain of the variable $d_{s}$ is $[0.50,2.00]$ and the required precision is two places after the decimal point. The required number of bits for a binary varible is computed by $2^{L_{5}-1}<2.00 \times 10^{2} \leq 2^{L_{5}}-1$. The length of a binary string for $d_{s}$ is $L_{5}=8$.

Therefore, the total length of a chromosome is $L=L_{1}+L_{2}+L_{3}+L_{4}+L_{5}=44$.

\subsubsection{Fitness calculation}

Chromosomes in a population evolve based on their fitness values. In this paper, unit production cost and unit machining time are to be minimized while profit rate is to be maximized. Therefore, profit rate should be converted to the following form in the fitness function

$$
P=\frac{1}{P_{t}}
$$

The fitness function is formed as follows:

$$
\text { fit }=w_{1} U C+w_{2} T C+w_{3} P .
$$

The objective functions with large values may dominate contribution of other objectives. To avoid this, Equation (16) is replaced with Equation (17),

$$
f i t=w_{1} \frac{U C}{U C_{\max }}+w_{2} \frac{T C}{T C_{\max }}+w_{3} \frac{P}{P_{\max }} .
$$

The fitness value is the sum of the three items and should be minimized. Therefore, a chromosome with a lower fitness value has a higher probability of being selected to survive.

\subsubsection{Crossover}

To avoid losing the best strings, $20 \%$ chromosomes with best fitness values in a population are selected to directly enter the new population. Crossover operations are performed on the rest $80 \%$ chromosomes. An integer from the range $[1,43]$ is randomly generated as the crossover point. Offspring is generated by exchanging the right parts of the two parent chromosomes. The new chromosomes through crossover are required to meet all constraints to the model. 


\subsubsection{Mutation}

Mutation rate is a probability to alter one gene (one bit of a chromosome). The mutation rate should be very low. Here the probability of mutation is set as 0.05 . After crossover, a number $r$ from $[0,1]$ is randomly produced and is compared with 0.05 . If $r \leq 0.05$, do mutation on that bit, changing zero to 1 or 1 to zero. During mutation operations, the created chromosomes are also required to meet all constraints. If some chromosomes do not, we keep creating new ones until the required number of satisfied chromosomes are generated.

\section{Case Study and Analysis}

The face-milling example given in Table 1 [11] is considered in this paper. Cemented carbide cutting tools are used to machine a gray cast iron workpiece (190HB). The same example was used to illustrate a solution approach [12].

Table 1. Data for the given example.

\begin{tabular}{l}
\hline$L=240 \mathrm{~mm}, D=160 \mathrm{~mm}, r_{e}=1 \mathrm{~mm}, B=100 \mathrm{~mm}, Z=16$ \\
\hline$k_{o}=0.5 \$ / \mathrm{min}, k_{t}=2.5 \$, t_{e}=1.5 \mathrm{~min}, t_{p}=0.75 \mathrm{~min}, S_{p}=25 \$, C_{m a}=0.5 \$$ \\
\hline$h_{1}=7 \times 10^{-4}(\mathrm{~min} / \mathrm{mm}), h_{2}=0.3(\mathrm{~min})$ \\
\hline$V_{\max }=300 \mathrm{~m} / \mathrm{min}, V_{\min }=50 \mathrm{~m} / \mathrm{min}, f_{\max }=0.6 \mathrm{~mm} / \mathrm{tooth}$, \\
$f_{\min }=0.1 \mathrm{~mm} / \mathrm{tooth}, d_{s, \max }=2 \mathrm{~mm}, d_{s, \min }=0.5 \mathrm{~mm}, d_{r, \max }=4 \mathrm{~mm}$, \\
$d_{r, \min }=1 \mathrm{~mm}$ \\
\hline$T=240 \mathrm{~min}, R_{s, \max }=0.0025 \mathrm{~mm}, R_{r, \max }=0.025 \mathrm{~mm}, F_{\max }=815.77 \mathrm{kgf}$, \\
$P_{\max }=10 \mathrm{~kW}, \eta=0.8$ \\
\hline$C_{v}=445, l=0.32, x_{v}=0.15, y_{v}=0.35, p_{v}=0, q_{v}=0.2, s_{v}=0.2, K_{v}=1.0$ \\
\hline$C_{f}=54.5, x_{f}=0.9, y_{f}=0.74, s_{f}=1.0, p_{f}=1.0, q_{f}=1.0, K_{f}=1.0$ \\
\hline
\end{tabular}

The optimization model was solved by the proposed GA approach with MATLAB programming for $d_{t}=$ 2.0, 2.5, 4.0, 6.0 and $8.0 \mathrm{~mm}$. Table 2 shows the optimal solutions and corresponding parameters by one computation for each $d_{t}$. The average values of unit production cost, unit machining time and profit rate after 20 repeats are given in Table 3 .

The results in Table 2 show that two rough passes and one finish pass are required when the total depth of cut is $d_{t}=8.0 \mathrm{~mm}$, with unit production cost of $1.3604 \$ /$ piece, unit machining time of $2.6296 \mathrm{~min}$, and profit rate of $8.7997 \$ / \mathrm{min}$. According to Ref. 12 , the unit production cost is $1.70 \$ /$ piece, unit machining time is $3.14 \mathrm{~min}$, and profit rate is $7.25 \$ / \mathrm{min}$. By comparison, the proposed optimization method reduces the unit production cost by $19.98 \%$ and the unit machining time by $16.25 \%$, and increases the profit rate by $17.61 \%$. The proposed method also presents better results than other methods in the literature [7].

Table 2. Optimal solutions and corresponding parameters by one computation.

\begin{tabular}{c|c|c|c|c|c}
\hline$d_{t}[\mathrm{~mm}]$ & 2.0 & 2.5 & 4.0 & 6.0 & 8.0 \\
\hline$d_{s}[\mathrm{~mm}]$ & 0.7118 & 1.2412 & 1.0941 & 0.8353 & 1.1000 \\
\hline$d_{r 1}[\mathrm{~mm}]$ & 1.2882 & 1.2588 & 2.9059 & 1.4113 & 2.9705 \\
\hline$d_{r 2}[\mathrm{~mm}]$ & - & - & - & 3.7534 & 3.9295 \\
\hline$f_{s}[\mathrm{~mm} /$ tooth $]$ & 0.2778 & 0.2556 & 0.2492 & 0.2810 & 0.2619 \\
\hline$f_{r 1}[\mathrm{~mm} / \mathrm{tooth}]$ & 0.5683 & 0.5286 & 0.4810 & 0.3063 & 0.5127 \\
\hline$f_{r 2}[\mathrm{~mm} / \mathrm{tooth}]$ & - & - & - & 0.3937 & 0.5683 \\
\hline$V_{s}[\mathrm{~m} / \mathrm{min}]$ & 227.167 & 235.103 & 272.161 & 268.55 & 259.40 \\
& 3 & 8 & 2 & 92 & 17 \\
\hline$V_{r 1}[\mathrm{~m} / \mathrm{min}]$ & 241.086 & 265.445 & 235.348 & 204.57 & 288.15 \\
& 7 & 7 & 0 & 88 & 63 \\
\hline$V_{r 2}[\mathrm{~m} / \mathrm{min}]$ & - & - & - & 182.29 & 266.54 \\
& & & & 55 & 46 \\
\hline$N$ & 2 & 2 & 2 & 3 & 3 \\
\hline$U C[\$ /$ piece $]$ & 1.0939 & 1.1001 & 1.0929 & 1.4440 & 1.3604 \\
\hline$T C[\mathrm{~min}]$ & 2.1049 & 2.1144 & 2.1042 & 2.7669 & 2.6296 \\
\hline$P_{t}[\$ / \mathrm{min}]$ & 11.1198 & 11.0671 & 11.1240 & 8.3327 & 8.7997 \\
\hline & & & & &
\end{tabular}

Table 3. Optimal solutions after 20 computations.

\begin{tabular}{c|c|c|c|c|c}
\hline$d_{t}[\mathrm{~mm}]$ & 2.0 & 2.5 & 4.0 & 6.0 & 8.0 \\
\hline$N$ & 2 & 2 & 2 & 3 & 3 \\
\hline $\begin{array}{c}U C \\
{[\$ \text { piece }]}\end{array}$ & 1.0781 & 1.0848 & 1.0806 & 1.3978 & 1.3922 \\
\hline$T C[\mathrm{~min}]$ & 2.0808 & 2.0916 & 2.0850 & 2.6922 & 2.6810 \\
\hline$P_{t}[\$ / \mathrm{min}]$ & 11.2573 & 11.1964 & 11.2334 & 8.5830 & 8.6217 \\
\hline
\end{tabular}

Our research demonstrates that GA operators have influence on the results of the objective functions. Tables 4-6 respectively show the variation of unit production cost, unit machining time, and profit rate with the change of crossover and mutation rates in our computational range, when the total depth of cut is $d_{t}=6 \mathrm{~mm}$ and the tool replacement time is $T=240 \mathrm{~min}$. The optimal unit production cost, unit machining time, and profit rate respectively take the best values of $1.3893 \$ /$ piece, $2.6767 \mathrm{~min}$, and $8.6355 \$ / \mathrm{min}$, all at the condition of crossover rate $=0.75$ and mutation rate $=0.04$.

Table 4. Unit production cost under various values of GA operators $\left(d_{t}=6 \mathrm{~mm}, T=240 \mathrm{~min}\right)$

\begin{tabular}{cccc}
\hline Crossover Rate & 0.6 & 0.75 & 0.8 \\
Mutation Rate & & & \\
\hline 0.03 & 1.4064 & 1.3987 & 1.3981 \\
0.04 & 1.3934 & 1.3893 & 1.3974 \\
0.05 & 1.3927 & 1.3939 & 1.3978 \\
\hline
\end{tabular}


Table 5. Unit machining time under various values of GA operators $\left(d_{t}=6 \mathrm{~mm}, T=240 \mathrm{~min}\right)$

\begin{tabular}{cccc}
\hline Crossover Rate & 0.6 & 0.75 & 0.8 \\
Mutation Rate & & & \\
\hline 0.03 & 2.7042 & 2.6918 & 2.6911 \\
0.04 & 2.6840 & 2.6767 & 2.6896 \\
0.05 & 2.6816 & 2.6840 & 2.6922 \\
\hline
\end{tabular}

Table 6. Profit rate under various values of GA operators $\left(d_{t}=6 \mathrm{~mm}, T=240 \mathrm{~min}\right)$

\begin{tabular}{cccc}
\hline Crossover Rate & 0.6 & 0.75 & 0.8 \\
Mutation Rate & & & \\
\hline 0.03 & 8.5421 & 8.5837 & 8.5859 \\
0.04 & 8.6099 & 8.6355 & 8.5910 \\
0.05 & 8.6185 & 8.6095 & 8.5830 \\
\hline
\end{tabular}

The objective function and some of the constraint functions in the optimization model are non-linear, using GA seems simpler than using conventional nonlinear optimization methods. Using those methods may require model linearization and approximation, and sometimes, with slow convergence.

\section{Conclusions}

The multi-objective optimization of machining parameters for face-milling operations was studied in this paper. Unit production cost, unit machining time, and unit profit rate were optimized simultaneously by Genetic Algorithms. The method presented in this paper can also be used in other machining operations such as grinding and drilling and some non-traditional machining processes. In addition, other objectives such as surface quality and tool life can also be optimized using the proposed method. These may form our future work in the area of machining parameter optimization. As well, Simulated Annealing (SA) and other metaheuristics may be used to solve these problems.

\section{Acknowledgements}

The research was jointly sponsored by the National Natural Science Foundation of China (51172062), the Scientific Research Foundation for the Returned Overseas Chinese Scholars from the Ministry of Education of China, the Natural Science Foundation of Hebei Province of China (E2012209017), and the Hundred Talents Program of Hebei Province of China (E2012100005).

\section{References}

1. F. Cus and U. Zuperl, Approach to optimization of cutting conditions by using artificial neural networks, Journal of Materials Processing Technology 173 (2006) 281-290.

2. Y. C. Shin and Y. S. Joo, Optimization of Machining Conditions with Practical Constrains, International Journal of Production Research 30 (1992) 2907-2919.

3. B. Gopalakrishnan and A.K. Faiz, Machine parameter selection for turning with constrains: an analytical approach based on geometric programming, International Journal of Production Research 29 (1991) 1897-1908.

4. R. Gupta, J. L. Batra, and G. K. Lal, Determination of Optimal Subdivision of Depth of Cut in Multipass Turning with Constrains, International Journal of Production Research 33 (1995) 2555-2565.

5. R. V. Rao, P. J. Pawar, Parameter optimization of a multi-pass milling process using non-traditional optimization algorithms, Applied Soft Computing 10 (2010) 445-456.

6. A. Jeang, H.-C. Li, Y.-C. Wang, A computational simulation approach for optimising process parameters in cutting operations, International Journal of Computer Integrated Manufacturing 23 (2010) 325-340.

7. R. Q. Sardinas, M. R. Santana, and E. A. Brindis, Genetic algorithm-based multi-objective optimization of cutting parameters in turning processes, Engineering Application of Artificial Intelligence 19 (2006) 127-133.

8. T.-S. Lan, M.-Y. Wang, Competitive parameter optimization of multi-quality CNC turning, Int $J$ Adv Manuf Technol 41 (2009) 820-826.

9. N. R. Abburi, U. S. Dixit, Multi-objective optimization of multipass turning processes, Int J Adv Manuf Technol 32 (2007) 902-910.

10. M. Solimanpur and F. Ranjdoostfard, Optimization of cutting parameters using a multi-objective genetic algorithm, International Journal of Production Research 47 (2009) 6019-6036.

11. W.A. Yang, Y. Guo, and W.H. Liao, Multi-objective optimization of multi-pass face milling using particle swarm intelligence, The International Journal of Advanced Manufacturing Technology 56 (2011) 429443.

12. N. Nefedov and K. Osopov, Typical Examples and Problems in Metal Cutting and Tool Design (Mir publishers, Moscow, 1987). 